\title{
An agent-based model to study compliance with safety regulations at an airline ground service organization
}

\author{
Alexei Sharpanskykh ${ }^{1} \cdot$ Rob Haest ${ }^{1}$
}

Published online: 28 May 2016

(c) The Author(s) 2016. This article is published with open access at Springerlink.com

\begin{abstract}
According to aviation statistics, most of the safety occurrences happen not in the air, but on the ground. Management of airlines and airports often consider failures to comply with safety-related regulations as important contributors to safety occurrences. To address the issue of compliance, approaches based on external regulation of the employees' behavior were proposed. Unfortunately, an externally imposed control is often not internalized by employees and has a short-term effect on their performance. To achieve a long-term effect, employees need to be internally motivated to adhere to regulations. To understand the role of motivation for compliance in ground service organizations, in this paper a formal agent-based model is proposed based on theories from social science with a wide empirical support. The model incorporates cognitive, social, and organizational aspects. The model was simulated and partially validated by a case study performed at a real airline ground service organization. The model was able to reproduce behavioral patterns related to compliance of the platform employees in this study. Based on the model, global sensitivity analysis was performed. The results of this analysis together with the simulation results were used to generate recommendations to improve compliance.
\end{abstract}

Keywords Compliance - Agent-based model ·

Motivation $\cdot$ Cognitive models $\cdot$ Social contagion

Alexei Sharpanskykh

o.a.sharpanskykh@tudelft.nl

1 Faculty of Aerospace Engineering, Delft University of Technology, Kluyverweg 1, 2629 HS Delft, The Netherlands

\section{Introduction}

Safety has the highest priority in aviation. Although commercial aviation is one of the safest modes of transportation, the ever increasing amount of traffic requires a substantial effort to decrease levels of risk. According to aviation statistics [6], most of the safety occurrences happen not during the flight, but on the ground, e.g. during aircraft ground handling operations and aircraft maintenance operations. Decreasing the number of ground safety occurrences has a high priority in many airlines in different countries. To achieve this aim some airlines use ramp Line Operations Safety Assessments (LOSA) [6] — a monitoring tool for measuring and identifying the adherence to safety regulations on the platform. Monitoring and recording of violations of safety regulations using ramp LOSA is done on a regular basis by experienced platform employees. It was expected that collecting information about violations and presenting this information to the employees would make them aware of their undesirable behavior. Unfortunately, the introduction of ramp LOSA at the ground service organization under study did not result in a decrease of the number of ground safety occurrences.

To address the issue of compliance of employees with safety regulations, an approach based on the STAMP framework was proposed in [5]. This approach appeals to topdown external control of the employees by management. The approach proceeds by identifying and mitigating deficiencies of an organizational control structure. However, as pointed out by [7], such an externally imposed control is often not internalized by humans and has a short-term effect on the performance of employees. To achieve a longterm effect, employees need to be internally motivated to adhere to regulations [7]. Thus, the main focus of this paper is on modeling and analysis of the motivation of platform 
employees to comply with safety regulations. Specifically, the following research questions are addressed:

- Which and how cognitive, social, and organizational factors influence the motivation of an employee to comply with safety regulations?

- Can a motivation model be developed to predict deviations from safety regulations in a real airline ground service organization?

- How and what kind of recommendations to improve compliance can be generated based on the analysis?

To answer these questions an agent-based motivation model was developed based on several theories from social science combined in an integrated framework. The model was applied in the context of a specific task of the aircraft arrival procedure - Foreign Object Damage (FOD) check. A foreign object is any object that should not be located near an aircraft, as it can damage aircraft or injure personnel. According to Boeing [4], the improper execution of FOD checks costs airlines and airports millions of dollars every year. Particularly the effects of FOD on maintenance costs, predominantly engine damage repairs, can be significant. Nevertheless, the ramp LOSA statistics showed that FOD checks are often not performed by platform employees.

The proposed model elaborates the motivation and decision making of the platform employees concerning whether or not to perform an FOD check. In this elaboration, individual cognitive, social, and organizational factors are taken into account. Furthermore, the model includes individual and social learning of agents representing the employees, and addresses two modes of reasoning of the agents explicit rational reasoning and implicit automatic reasoning (habits). To initialize the model, an extensive one year study was performed at a real ground service organization. Data were gathered by observation, questionnaires, and interviews with employees playing different roles in the organization. The collected data were separated into two data sets. The first set contained data on the organizational context (i.e., formal organizational structures and processes, norms, and regulations) and on local processes and characteristics of the organizational agents. This dataset was used to initialize the model. To represent the uncertainty and variability of the components of the model, most of the parameters were specified by intervals with a uniform distribution. The second set contained data describing global organizational or systemic properties (such as ramp LOSA statistics), which were used to validate the model. Some of the model validation results are discussed in the paper. The model was able to capture behavioral patterns of the platform employees and reflect the ramp LOSA statistics concerning FOD check compliance in the real ground service organization.

Based on the model, global sensitivity analysis was performed to identify the parameters with the highest impact on compliance. The information about these parameters, combined with the simulation results, was used to formulate recommendations to improve compliance. These recommendations are summarized in the paper.

The paper is organized as follows. In Section 2, the theoretical basis of the model is described. In Section 3, the proposed agent-based model is provided. The main results from the simulation study are discussed in Section 4. Section 5 presents the results of the sensitivity analysis. Recommendations to improve compliance are discussed in Section 6. The paper ends with conclusions and a discussion of the results.

\section{Theoretical background}

The theoretical basis of the model comprises several theories from social science, which are summarized below. These theories address universal human needs, the way humans reason about their needs, and how they make choices to act based on this reasoning. All the theories used for the model development have strong empirical support.

Self-determination theory [7] is a theory of human motivation, which addresses people's universal, innate psychological needs and tendencies for growth and fulfillment. Specifically, the theory postulates three types of basic needs:

- the need for competence concerns the people's inherent desire to be effective in dealing with the environment;

- the need for relatedness concerns the universal disposition to interact with, be connected to, and experience caring for other people;

- the need for autonomy concerns people's universal urge to be causal agents - to have volition.

In line with other motivation theories [22], in addition to the needs listed above, the need for safety was added, which is particularly relevant for the ground service organization, in which physical injuries are not uncommon.

Based on needs, individual goals can be defined. Higher level individual goals may be refined in goal hierarchies as described in [23]. A goal is a state that the individual desires to achieve or maintain. To achieve or maintain his or her goals, an individual considers different behavioral options (actions or plans). One of the theories that explains why individuals choose one option over another 
is the Expectancy Theory of Motivation by Vroom [22]. Advantages of the Expectancy Theory include: (a) it can be formalized; (b) it allows incorporating the organizational context; (c) it has received good empirical support. According to this theory, when an individual evaluates alternative possibilities to act, he or she explicitly or implicitly makes estimations for the following factors: expectancy, instrumentality, and valence.

Expectancy refers to the individual's belief about the likelihood that a particular act will be followed by a particular outcome (called a first-level outcome). Its value varies between 0 and 1 .

Instrumentality is a belief concerning the likelihood of a first level outcome resulting into a particular second level outcome; its value varies between -1 and +1 . Instrumentality takes negative values when a second-level outcome has a negative correlation with a first-level outcome. A second level outcome represents a desired (or avoided) state of affairs that is reflected in the agent's goals.

Valence refers to the strength of the individual's desire for an outcome or state of affairs; it is also an indication of the priority of goals.

Values of expectancies, instrumentalities and valences may change over time, in particular due to individual and social learning. The motivational force of an individual $i$ to choose option to act $k$ is calculated as:

$F_{k, i}(t)=\sum_{l=1}^{n} E_{k l, i}(t) \sum_{h=1}^{m} V_{h, i}(t) I_{k l h, i}(t)$

Here $E_{k l, i}(t)$ is the strength of the expectancy that option $i$ will be followed by outcome $j ; V_{h, i}(t)$ is the valence of the second level outcome (a goal) $h$; and $I_{k l h, i}(t)$ is the perceived instrumentality of outcome $l$ for the attainment of outcome $h$ for option $k$.

The Vroom's theory describes the process of rational decision making. However, repetitive actions such as those that occur during aircraft handling may over time become automatic, i.e., a habit. The dual process theory [16] distin- guishes System 1 and System 2 thinking. While System 2 is used for rational, rule-based, and analytic thinking, System 1 is associated with unconscious, implicit, and automatic reasoning. Depending on the dynamics of environmental changes, an individual switches between the systems. Both systems are used in the model and the case study considered in the paper.

In the following section, we demonstrate how the theories from this section were formalized and integrated into an agent-based model.

\section{The agent-based model}

To develop the model, the steps of a generic methodology for modeling agent organizations from [26] were used. In what follows, these steps are elaborated.

\subsection{Identification of organizational roles}

The following roles were identified (Fig. 1): Platform Employee, Team Leader, and Sector Manager. The Platform Employee and Team Leader roles form a composite Team. For each Team, four Platform Employee role instances and one Team Leader role instance are specified. There is one Sector Manager role instance in the model.

\subsection{Identification of interactions among roles and with the environment}

All roles are able to interact with each other (Fig. 1). The Platform Employee role and Team Leader role are able to observe the tasks to be accomplished in the environment (i.e., aircraft to be handled), outcomes of their own actions (task is finished successfully, task duration, a safety occurrence happened during the task), and task execution by the other Platform Employee agents. Furthermore, the Team Leader and Sector Manager roles are able to observe violations of the procedures and to provide reprimands to the
Fig. 1 The role instances and interactions among them in the model

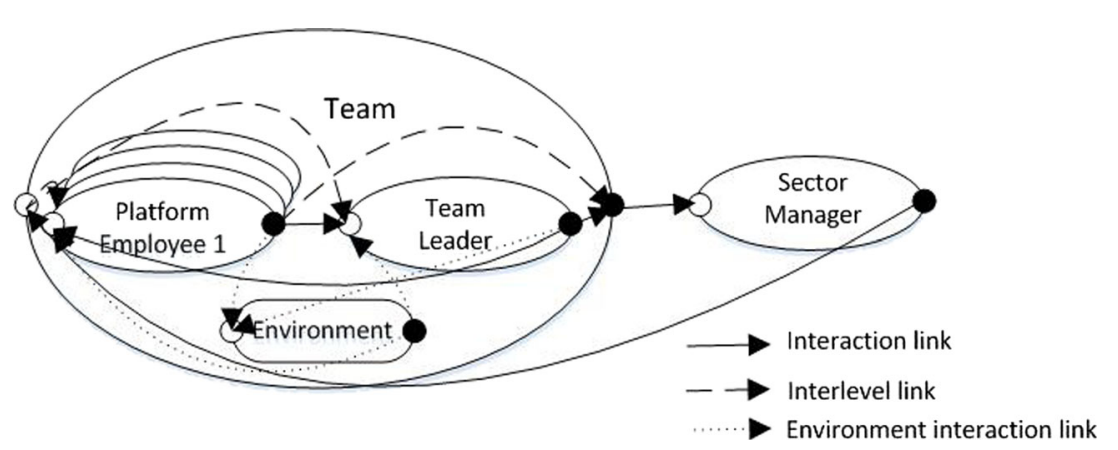


Platform Employee roles. The reprimands can be observed by all roles.

\subsection{Identification of tasks and workflows}

Each Team role handles one aircraft at a time. Aircraft to be handled are organized in a FIFO queue. The specific focus of this study is on the FOD check task in the aircraft handling procedure. There are several reasons why this task was chosen. First, the FOD check task is an essential part of the aircraft arrival procedure, which is performed many times during a day by different platform employees. Second, the improper execution of FOD checks costs airlines and airports millions of dollars every year. Third, aviation statistics show that among all the tasks of the arrival procedure, FOD checks are most often not performed by platform employees. Fourth, this task involves individual decision making of platform employees, social processes, and formal organizational aspects, such as formal authority and formal procedures. Therefore, this task is well suited to answer the research questions of the paper. Furthermore, this task is performed separately from other tasks of the arrival procedure, and differs from these tasks substantially. Thus, to analyze the execution of FOD checks, other tasks can be modeled at a higher level, represented by time intervals based on the operational statistics from the organization under study. The sensitive raw data cannot be presented in the paper for confidentiality reasons. Note that individual decision making and the social mechanisms modeled in this paper are based on general psychological and social theories and are not restricted to the FOD check task only.

Specific agents are allocated to the role instances, which in contrast to roles and role instances may have internal cognitive states and dynamics, which are described in the following steps.

\subsection{Identification of characteristics of agents}

Platform Employee agents have three characteristics: risk aversion (reflected in parameters of the expectancy theory model E13, E14, E15, E16, I131, I141, I152, I162, in Fig. 2, openness to new experiences, and expressiveness in communication. All of these characteristics are assigned values in the interval $[0,1]$.

\subsection{Identification of goals and needs of agents}

In accordance with self-determination theory and the field study, a number of goals and subgoals were identified in Table 1). The types of needs identified in Section 2 were used by ethnographers to elicit the goals of the platform employees, who participated in the study. The goals were elicited by interviews with the platform employees and their
Fig. 2 Decision making model of a Platform Employee agent for performing FOD check based on the Expectancy Theory with expectancies $(\mathrm{E})$, instrumentalities (I), states (S), and valences $(\mathrm{V})$. For readability purposes the time parameter and agent indexes have been omitted

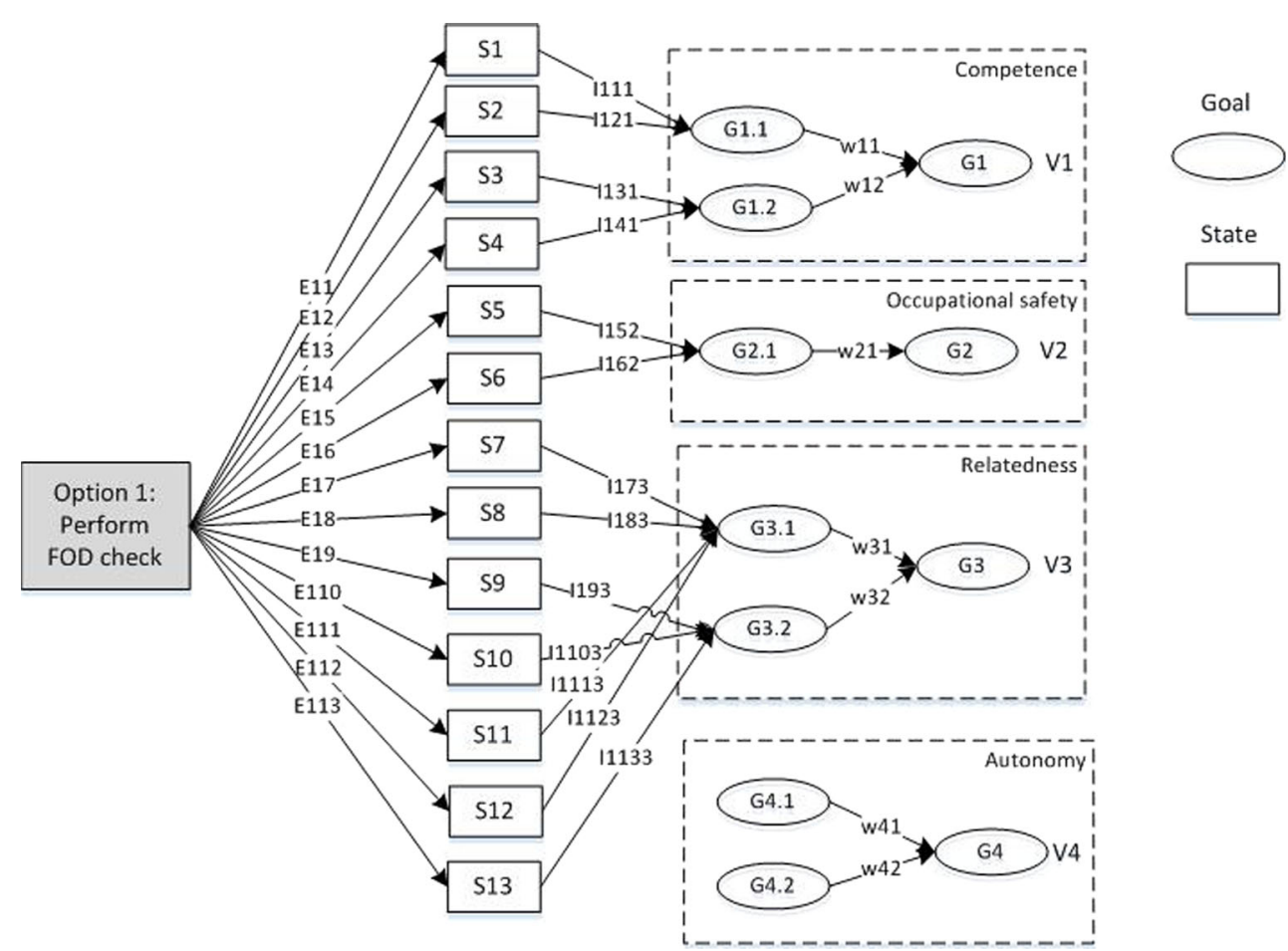


Table 1 The goals and states of the decision making model provided in Fig. 1

\begin{tabular}{ll}
\hline Goals & States \\
\hline G1 Achieve a high level of competence & S1 Action saves time \\
G1.1 Achieve the highest time efficiency & S2 Action costs additional time \\
G1.2 Prevent aircraft, equipment, and/or & S3 Action results in aircraft, equipment, or \\
infrastructural damage & infrastructural damage \\
G2 Achieve a high level of occupational & S4 Action prevents aircraft, equipment, or \\
safety & infrastructural damage \\
G2.1 Prevent personal injury & S5 Action results in personal injury \\
G3 Maintain sense of belonging and & S6 Action prevents personal injury \\
attachment to colleagues & S7 Action is in alignment with the team \\
G3.1 Maintain high team acceptance & member norms \\
G3.2 Maintain high management acceptance & S8 Action is not in alignment with the team \\
G4 Achieve a high control over own & norms \\
behavior and goals & S9 Action is in alignment with sector \\
G4.1 Achieve a high level of freedom in the & management norms \\
execution of tasks & S10 Action is not in alignment with sector \\
G4.2 Achieve high psychological ownership & management norms \\
of rules & S11 Reprimand received from team member \\
& S12 Reprimand received from team leader \\
& S13 Reprimand received from sector \\
& manager \\
\hline &
\end{tabular}

managers, and based on observation of their daily practices. For each type of need, questions were prepared to refine goals based on the need in the specific context of the case study. Furthermore, the priorities of the goals were established. A detailed description of the procedure, as well as of the obtained outcomes, is provided in [3, 10, 14]. For more details on the identified goals, please refer to Appendix A.

\subsection{Identification of beliefs of agents}

All the expectancies, instrumentalities, and valences of the Platform Employee agents are represented by their beliefs. Thus, a numerical value is associated with every agent's beliefs.

\subsection{Specification of decision making of agents}

Decision making by the Platform Employee agents about whether or not to perform an FOD check was modeled by using the Vroom's expectancy theory (Fig. 2). To initialize the expectancies, instrumentalities, and valences of the model for each agent, three classes of values were introduced: Low, Medium, and High. This was done to address the issue of uncertainty in these parameters, and individual variations of the platform employees. Most of the numerical scales of these parameters were divided equally among the classes: Low for [0, 0.33), Medium for [0.33, 0.67), and High for $[0.67,1]$. For a few scales, the division among the classes was adjusted depending on the interpretation of the corresponding parameters in the context of the case study. To determine the values (i.e., specific classes) of the parameters, an ethnographic study was performed in the real ground service organization by observation of organizational practices, interviews, and questionnaires with employees. Furthermore, secondary sources were used, such as safety reports, safety statistics, and reports on previous operational studies at the organization. The complete list of the parameter values of the model, and justifiations for the choices of their values, are provided in Appendix B.

The expectancy theory model was used for System 2 reasoning. When the same operations were routinely executed by a Platform Employee agent, the agent's System 2 reasoning was gradually shifting to System 1 reasoning - a habit had been formed. This shift was modeled by the dynamics of agent $i$ 's openness parameter $\alpha_{i}$ :

$\alpha_{i}(t+\Delta t)=\alpha_{i}(t)+\zeta\left(\alpha_{i}^{\min }-\alpha_{i}(t)\right) \Delta t$,

where $\alpha_{i}^{\min }$ is the minimum perceptive openness of agent $i$ (set to 0.1 in the simulation), and $\zeta$ is the rate of transition from System 2 to System 1. It depends on the execution frequency of the operation by the agent, as well as on 
the agent's personal characteristics. In the simulation $\zeta=$ 0.015 , meaning that it takes around two months to form a new habit.

When procedural rules change, an agent needs to adapt to a new situation and reconsider options by switching from System 1 to System 2: the agent $i$ 's openness is set to its initial value $\alpha_{i}(0)$, and the process of the new habit formation starts again.

A similar expectancy theory model was created for option 2 - 'Not to perform FOD check'. It has the same types of parameters, but their values are different (see Appendix B).

In the simulation, every time an agent $i$ considers explicitly (System 2) or implicitly (System 1) whether or not to perform an FOD check, motivation forces $F_{1, i}$ and $F_{2, i}$ for both options are calculated by (1). Then, the agent performs the FOD check with probability $\left(F_{\max }+F_{1, i}\right) /\left(2 F_{\max }+\right.$ $\left.F_{1, i}+F_{2, i}\right)$. The normalization with $F_{\max }$ is used to compensate for the negative values of the instrumentalities.

\subsection{Specification of agent learning and social interaction}

Two types of learning were modeled: individual and social learning of agents.

An agent learns individually by observing a feedback from the environment on its action. In the decision making model from Fig. 1, the individual learning was realized by updating values of expectancies (E) based on the following observations:

- An agent observes whether or not a reprimand from other agents is provided, when the agent does not comply with regulations (update of E111, E112, E113).

- After the successful execution of a task, an agent observes how much time it took and how it influenced the total execution time of the operation (update of E11, E12). The task durations were determined based on operational data from the organization under investigation.

- When an agent does not perform a FOD check, a safety occurrence could occur. The agent is able to observe such occurrences (update of E13, E14). For this also the organizational statistics was used.

Furthermore, the Platform Employee agents are able to observe the execution of operations by other agents in their teams, and to learn from these agents by verbal communication. Social learning is modeled as the process of social contagion [13]. By this process, expectancies $E_{k l, i}(t)$ were updated as:

$E_{k l, i}(t+\Delta t)=E_{k l, i}(t)+\delta_{k l, i}(t) \Delta t$
Here

$\delta_{k l, i}(t)=\sum_{j \in T} \gamma_{j, i}(t)\left(E_{k l, j}(t)-E_{k l, i}(t)\right) / \sum_{j \in T} \gamma_{j, i}(t)$

is the amount of change of the agent $i$ 's state; $T$ is the set of the agents in the team. A weight $\gamma_{j, i} \in[0,1]$ is the degree of influence of agent $j$ on agent $i$ defined as:

$\gamma_{j, i}(t)=\alpha_{i}(t) \varepsilon_{j}(t) \beta_{j i}$

$\alpha_{i}(t)$ and $\varepsilon_{j}(t)$ are the agent characteristics - the openness of information recipient agent $i$ and the expressiveness of information provider agent $j$, and $\beta \in[0,1]$ is the strength of the information channel between the two agents.

The communication style in the teams of platform employees is direct, informal, and of a high frequency. For direct communication between agents $\beta_{j i}=1$.

Sector Managers communicate with the Platform Employees directly during observation tours and dedicated meetings. Indirect communication with the management occurs by messages on information screens, posters, memos etc. $\left(\beta_{j i}=0.3\right)$. The expectancy values of the agents are updated once per simulated day.

\subsection{Identification of shared beliefs, norms and values of (groups of) agents}

By field observations and interviews, a team norm was identified. The norm applies to situations in which a team arrives too late at an aircraft stand while the aircraft is waiting for the docking process. To save time, the FOD check is omitted and the arrival procedure starts directly. Field data revealed that employees who execute the check in the described situation get a social reprehension from other team members. This influences the achievement of goal G3.1, which is driven by the alignment of the decision option with the team norms and team leader norms.

Expectancy of agent $i E_{17, i}(t)$ that decision option 1 is in alignment with the team norms depends on the degree of similarity between decision option 1 and the most relevant team norm (if one exists, otherwise $E_{17, i}(t)=0$ ) and the likelihood that agent $i$ is familiar with this norm. The value of corresponding $I_{173}$ is determined as the product of the perceived importance of the norm in the team and the degree of connectedness of agent $i$ in the team: $\sum_{j \in T, i \neq j}\left(\gamma_{i j}+\gamma_{j i}\right) / 2(|T|-1)$, where $T$ is the set of agents in the team. Intuitively, to have a large positive contribution to team relatedness goal G3.1, the agent $i$ 's decision option should be in line with an important team norm, and agent $i$ should be well-connected in the team, i.e. be its prototypical member. This way of reasoning is inspired by prototypicality theories [21]. 
The Team Leader agent's norms and Sector Manager agent's norms are in line with the organizational regulations.

\subsection{Specification of the environmental dynamics}

The number of aircraft to be handled by the teams of platform employees was modeled according to the actual operational statistics. A day was divided into three shifts (morning, afternoon/evening, and night). During normal operations, on average, the arrival procedure is executed three times each shift.

\section{Simulation study}

In this section, first a simulation setup is described (Section 4.1), then simulation results are discussed (Section 4.2)

\subsection{Simulation setup}

Here we discuss a scenario that occurred in reality and that could be validated by empirical data from ramp LOSA. In this scenario, three periods of the organizational operation are considered:

- the first period, with limited managerial control over the execution of the platform operations and limited safety information provision;

- the second period (8 weeks), with high managerial control after many safety occurrences happened in the first period;

- the third period, in which the release of managerial control occurs over time (a linear transition from High control to Low control mode, Table 2).

The parameters for these periods, which were determined by using the field study data, are provided in Table 2 . The organization under study does not collect data about reprimands; the probabilities in Table 2 were derived from interviews. In general, the platform employees knew that the reprimands were not likely to be provided, which was in line with the culture of the organization. Therefore, even in the High control mode, the probabilities of reprimands were not high. In the Low control mode, the activities of the platform employees were rarely observed by the management, and violations of procedures were often tolerated. Furthermore, weak social control with respect to safety was realized within the teams. The coercive power of both the sector manager and the team leaders was low. In the High control mode, the team leaders were largely held responsible for observing compliance of the platform employees with regulations and procedures. The sector manager could observe on average three times less operations than the team leaders in the High control mode.

In the Low control mode, very little communication of safety-related information occurred in the organization, which is reflected in the parameter settings in Table 2. In the High control mode, communication has significantly improved. The safety manager organized intensive communication sessions, as well as communicated with platform employees on the workfloor. Furthermore, diverse indirect communication means were employed, such as posters, newsletters, and memos, to remind and inform the platform employees about safety regulations. However, the expressiveness of such information sources was perceived by the platform employees as lower than the expressiveness of management in direct communication.

A team consists of 5 agents: a Team Leader and 4 Platform Employees. In the simulation, the agents in the teams communicated with each other in a random order.

According to interviews at the organization under study, two types of agents in the teams were identified: more expressive agents with $\varepsilon_{i} \in[0.5,0.9]$, and less expressive agents with $\varepsilon_{i} \in[0.1,0.5]$. Each agent can be of either type with an equal probability. The openness of an agent $\alpha_{i}$ was assigned a wide range [0.1, 0.9], to represent the diversity of agents. In each simulation run, the agents' parameters were randomly generated using the uniformly distributed intervals introduced above and in Appendix B.

In the simulation, every time step corresponds to a decision moment for executing or not executing the FOD check. One simulation day is divided into three shifts (morning, afternoon/evening, and night). During normal operations, on
Table 2 The parameters of the managerial control modes used in the simulation

\begin{tabular}{lll}
\hline Parameter & Low control & High control \\
\hline Probability of reprimand from Sector Manager agent & 0.05 & 0.11 \\
Probability of reprimand from Team Leader agent & 0.05 & 0.33 \\
$\varepsilon_{S M}(t)$ in direct communication & 0.06 & 0.6 \\
$\varepsilon_{S M}(t)$ in indirect communication & 0.03 & 0.3 \\
\hline
\end{tabular}




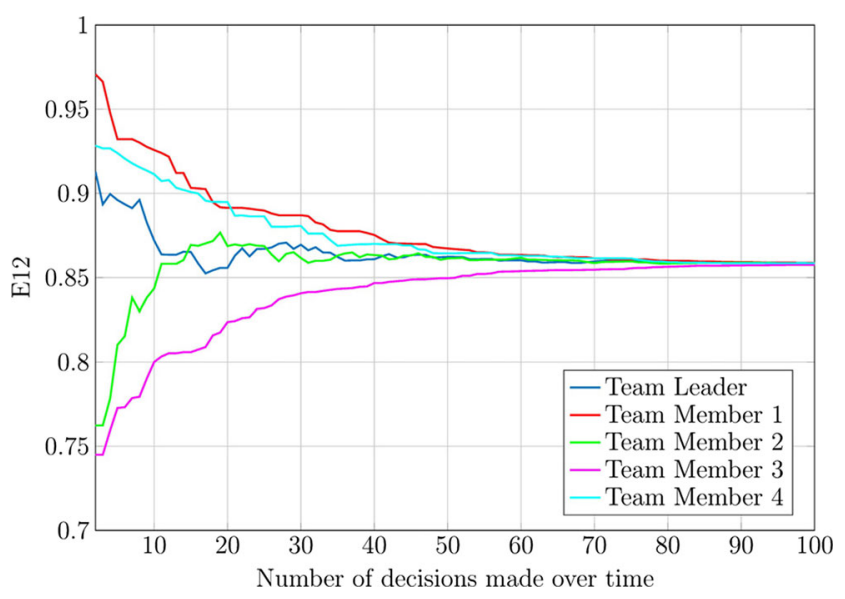

Fig. 3 Dynamics of expectancy $E_{12}(t)$ - belief about the likelihood that the execution of the FOD check would cost additional time - for all agents in a team

average, the arrival procedure is executed three times each shift. The simulated time period was 200 working days; $\Delta t=1 / 3$, indicating that on average 3 decisions are made by each Platform Employee agent per day.

\subsection{Simulation results}

In this section, the main simulation results obtained for the scenario under consideration are described.

Due to frequent observation and communication of the agents in a team, their expectancies related to the exchanged information tend to converge over time (Fig. 3). This is a well-known effect in the literature on social contagion $[1,13]$.
Simulation experiments with a varying composition of teams indicated that the individual characteristics of the Team Leader agent play a crucial role in the dynamics of social contagion, and largely determine expectancies in a team.

Specifically, consider simulation results for two particular teams (Table 3), which exemplify desired and undesired effects of social learning (Fig. 3). In both teams information concerning parameter E22 (belief about the likelihood that performing FOD check would prevent aircraft damage) is being exchanged between the agents.

High values of parameter E22 are desired, since it indicates the degree of safety awareness of a Platform Employee agent. In Team 1 the team leader has a high value for parameter E22 and a high expressiveness. The team members have a high openness. In such a team, the E22 values of the agents change in a desired way, i.e., converge to a high value, close to the initial value of the team leader.

Social contagion can also result in undesired dynamics of expectancy values, as in the case with Team 2 (Table 2). In this example, Platform Employee agents 7 and 8 have a high expressiveness and risk-taking attitude, which in combination influence negatively the expectancies of Platform Employee agents 5 and 6 . The team leader has a limited influence on the team members to prevent this undesired process (Fig. 4).

Both desired and undesired dynamics, as was studied by the simulation, were recognized by sector managers in the organization under study. They observed that the compliance with regulations and overall performance vary much over the teams. Furthermore, it was confirmed that negative social influence is not limited to single teams but spreads
Table 3 The characteristics of the agents in two particular teams used to examine the dynamics of social contagion

\begin{tabular}{llll}
\hline Agent & Risk aversion & Openness & Expressiveness \\
\hline Team 1 & & & \\
Team Leader 1 & H & L & H \\
Platform Employee 1 & M & H & L \\
Platform Employee 2 & M & M & L \\
Platform Employee 3 & L & M & L \\
Platform Employee 4 & L & M & L \\
Team 2 & & & \\
Team Leader 2 & H & L & L \\
Platform Employee 5 & M & M & L \\
Platform Employee 6 & M & M & L \\
Platform Employee 7 & L & L & H \\
Platform Employee 8 & L & L & H \\
\hline
\end{tabular}

Each parameter is classified as High $(\mathrm{H})[0,0.33)$, Medium $(\mathrm{M})[0.33,0.67)$ or Low $(\mathrm{L})[0.67,1]$ 

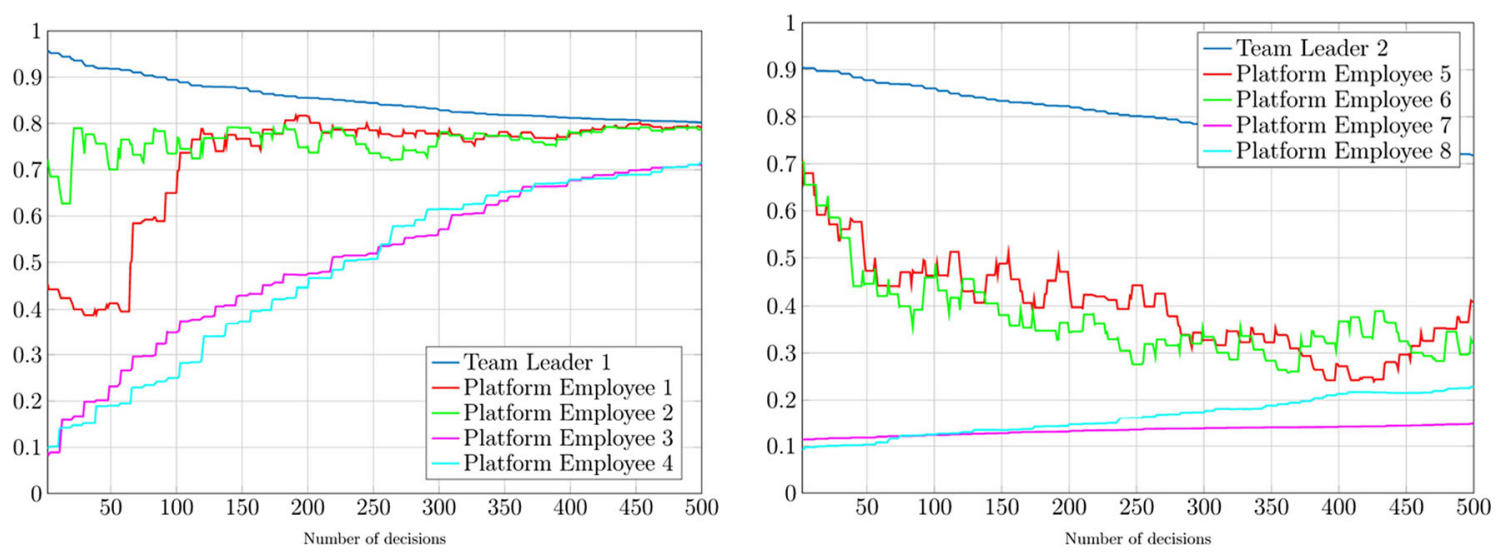

Fig. 4 The change of E22 (belief about the likelihood that performing FOD check would prevent aircraft damage) of the agents in two teams described in Table 2

over the whole organization. According to the sector managers, the findings are not limited to safety-related attitudes, but also concerns operational performance of employees.

In the following we discuss simulation results for the scenario described in Section 4.1.

In the first period of the simulated scenario, after the initialization phase, most of the agents have a relatively constant motivational force for both decision options (Fig. 5). The motivational forces to perform FOD checks are low, as the organization neither sufficiently controls the execution of operations nor creates a sufficient awareness about the importance of FOD checks. Some agents in the team even prefer not to perform the check. By the end of this phase, the agents function in System 1 mode of reasoning.

In the beginning of the second period (indicated by the dotted vertical line in Fig. 5) the organization introduces more frequent managerial control and reprimands. To adapt to the new circumstances, the agents switch to a System 2 mode of reasoning. Such a change results in an increased motivation to perform FOD checks and a decreased motivation not to do so of all agents in all teams. The differences in motivation are explained by differences in the individual characteristics of the agents. However, when after 8 weeks the control and information provision was gradually removed, the agents start gradually returning to their previous state. This form of motivated behavior is known in the literature as 'externally regulated behavior' [7]. Such a form of motivation is sustained by the continuous presence of reprimands and rewards. These behavioral patterns were also observed in the ground service organization under study.

Model variants with larger shifts of 7 and 10 agents were also simulated. The patterns in Fig. 5 were also observed in these cases (Appendix D). However, the more agents that participated in a shift, the higher were the motivational forces both to report and not to report. This can
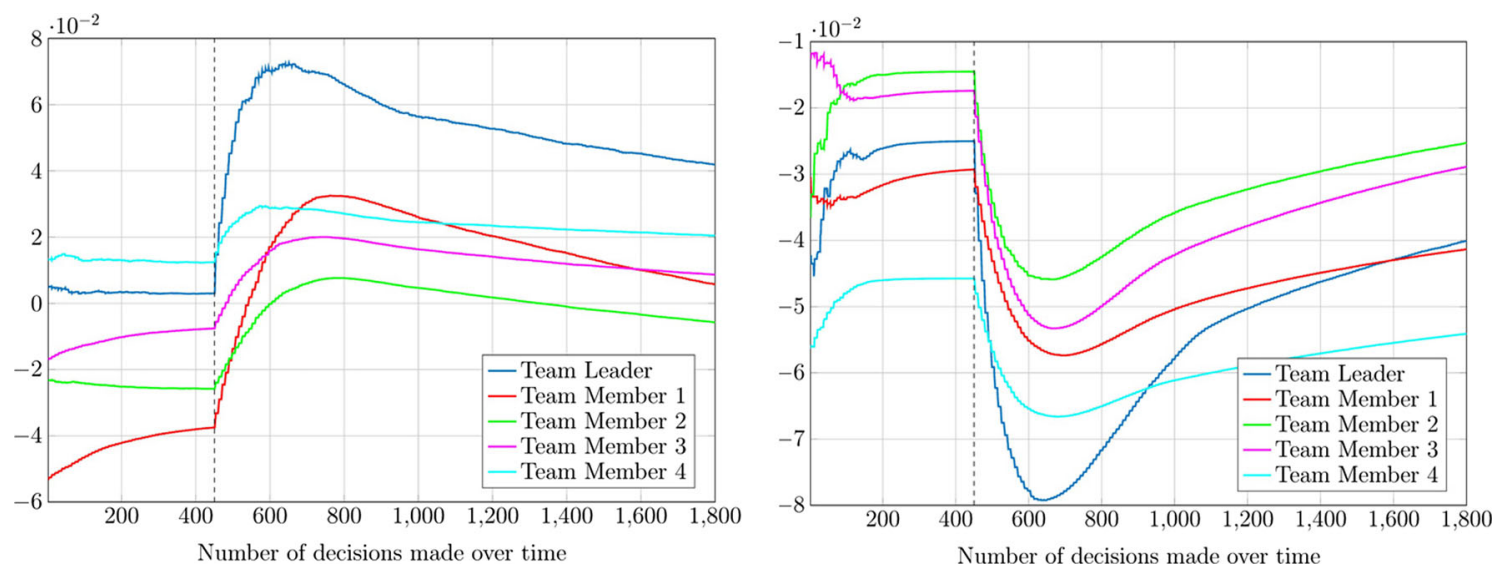

Fig. 5 The motivational forces of 5 agents in a team to perform FOD check (left) and to not perform FOD check (right) in the three periods of the scenario under consideration. The dotted vertical line indicates the beginning of the second period (increased control) 
be partially explained by more intense social interaction and influence processes in a shift. Furthermore, clusters of agents demonstrating similar behavior emerged in larger shifts, which were particularly well distinguishable in shifts with 10 agents. In the teams, in which all members are expected to interact intensively with each other, it might be an undesirable property.

The model simulation outcomes were also compared to the ramp LOSA statistics of the FOD checks execution in the ground service organization (Fig. 6). The model was able to capture the trends in real ramp LOSA data. Specifically, in Q2, characterized by limited managerial control and limited information provision, both the simulated outcomes and real data indicate a low percentage of the executed FOD checks. In Q3, characterized by a substantial increase of managerial control, a rapid increase in the percentage of executed FOD checks is observed, both in the simulated outcomes and real data. In Q4, a high percentage of the executed FOD checks persists. Starting from Q4, strict managerial control is gradually released. This results in a decrease of the percentage of the executed FOD checks, as can be seen in both simulated and real data for Q1.

The simulation outcomes presented in Fig. 6 fit a normal distribution. Since trace empirical data for the whole simulation were not available in this study, the validation of the model outcomes was done using Student's two-sample t-test, as also recommended in [17]. Student's two-sample t-test performed on real and simulated data supported the null-hypothesis at the significance level $5 \%$.

\section{Sensitivity analysis}

In this section, a sensitivity analysis approach is described in Section 5.1. Then, the sensitivity analysis results are presented in Section 5.2.

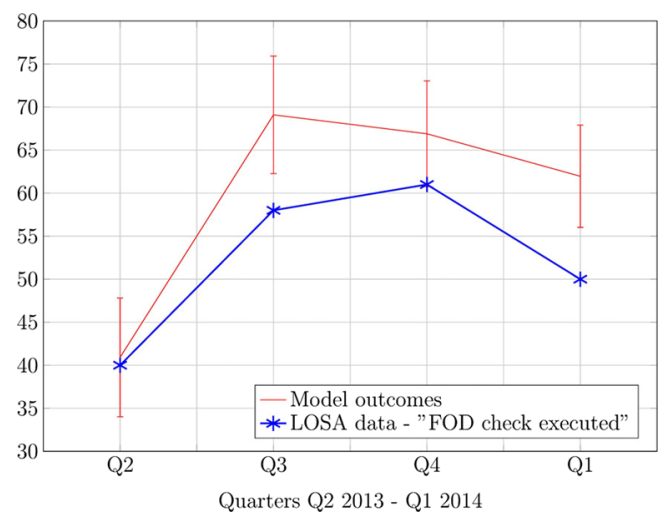

Fig. 6 The FOD check execution statistics (in \%) obtained from the ramp LOSA data and from the simulated model. The model outcomes are the mean and standard deviation values of simulation per quarter calculated over 3000 simulation runs

\subsection{Sensitivity analysis approach}

Based on the model, sensitivity analysis was performed by using the Monte Carlo filtering method [25]. This is a global sensitivity analysis method, which has the advantage over local methods that the analysis is not limited to changing one parameter at a time while keeping the other parameters fixed. The aim of Monte Carlo filtering is to identify model parameters variation of which according to associated credibility intervals leads to significant differences in the model output. It consists of two steps, which are presented next.

\section{Step 1: MC simulations}

For the complete set of model parameters, lower and upper bounds of credibility intervals of their values were determined based on data gathered at the organization under investigation and on our knowledge about the uncertainty in modeled aspects (Appendix B). Next, 3000 Monte Carlo simulation trials were performed where in each simulation the parameters were chosen uniformly within their credibility interval bounds. For each input factor $x_{i}$ two sets of values were determined: $x_{i} \mid F$, containing all values of $x_{i}$ from the simulations that produced the acceptable motivation force levels, and $x_{i} \mid \underline{F}$, containing all $x_{i}$ values that produced unacceptable (low) motivation force levels The threshold that separated the acceptable values from the unacceptable ones was set as the 0.3-percentile of the empirical probability distribution of the motivational force to perform FOD check obtained by the Monte Carlo simulations.

Step 2: Smirnov test

A Smirnov two sample test was performed for each input factor independently. The applied test statistics are

$d\left(x_{i}\right)=\sup \left\|P_{F}\left(x_{i} \mid F\right)-P_{F}\left(x_{i} \mid \underline{F}\right)\right\|$,

where $P_{F}$ and $P_{F}$ are marginal cumulative probability distribution functions calculated for the sets $x_{i} \mid F$ and $x_{i} \mid \underline{F}$, respectively. A low level of $d\left(x_{i}\right)$ supports the nullhypothesis $H_{0}: P_{F}\left(x_{i} \mid F=P_{F}\left(x_{i} \mid \underline{F}\right)\right.$, meaning that the parameter $x_{i}$ is not important, whereas a high level of $d\left(x_{i}\right)$ implies the rejection of $H_{0}$, meaning that $x_{i}$ is a key factor.

To investigate the effects of the credibility intervals on the sensitivity analysis results, the sensitivity analysis procedure was also performed with wider ranges of the parameters of the decision making model reflecting a larger diversity of the Platform Employee agents and their attitudes than was identified in the organization under study. Specifically, the expectancies, instrumentalities, and valences varied in the whole range $(0,1)$. 


\subsection{Sensitivity analysis results}

In the following sections, the main findings concerning different types of parameters of the model are discussed. The sensitivity analysis findings obtained by the MC simulation with the parameter ranges defined in Appendix B mostly agreed with the findings obtained by the MC simulation with wider ranges as defined in Section 5.1. The only difference is that in the case with the wide parameter ranges, the parameters related to the reprimands from the team and the team leader were identified as important. This is because the simulation settings allowed formation of diverse attitudes towards reprimands, which had a significant impact on whether or not to perform the FOD check task. A complete overview of the sensitivity analysis results is provided in Appendix C.

Instrumentalities The importance of instrumentalities in the model is considerably larger than the importance of expectancies, weights, and valences. The instrumentalities answer the question why a decision option considered by an agent is important for the achievement of its goals. Also, according to other studies (e.g., in incident management and emergency [20]), explaining why an action needs to be performed has a higher motivational effect on the agent than simply providing information or instructions.

Time perceptions Analysis revealed that the parameters related to the perception of time efficiency $\left(E_{12}, I_{121}\right)$ have a large impact on the model outcomes. This was also recognized by the domain experts.

Risk perceptions In the model, the perceived effectiveness of the FOD check for preventing (engine) damage ( $E_{22}$ and $I_{22}$ ) was important.

Connectedness and influence relations Social relations within teams and with the management (I411b and I431b) have a major contribution to individual decision making and significantly impact the model outcomes. This was also recognized by the domain experts.

Norms The parameters related to the team norms (E411a and I411b) have a high influence on the model outcomes.

The importance of the autonomy-related variables $I F_{1}$ (Amount of monitoring and control) and $I F_{2}$ (Level of rule details) is comparable with the importance of the expectancies.

Valences As expected, the importance of the competence valence $\left(V_{1}\right)$ was considerably larger than of the other valences.
Management reprimands In contrast with what was expected, the importance of managerial reprimands (E53 and I53) is relatively low compared with other parameters. This is primarily because of the low probabilities of reprimand provision by the Sector Manager agent (Just culture). Together with a relatively low reprimand severity, the effect of management influence through control is low.

\section{Recommendations}

Based on the sensitivity analysis results discussed in Section 5, a number of recommendations for improvement of compliance were formulated, which were divided into four areas: information provision, managerial control and influence social interaction in teams, and autonomy.

\subsection{Information provision}

Provision of information (through formal and informal communication channels) is performed by the management to change safety-related beliefs and attitudes of the employees. Simulations revealed that information provision positively affects time and risk perceptions (expectancies) of the Platform Employee agents which contributes significantly to the desired motivational forces. The effectiveness of information provision depends on the strength of information channels Interviews with managers and platform staff revealed that the information screens in the canteen and direct communication (e.g. information sessions) are effective channels. The effectiveness of sending emails and workplace instructions is limited. The impact of magazines like Ground Safety is questioned. Direct communication between sector managers and platform staff seems to be the most adequate way of influencing employees. It is recommended that higher management should facilitate sector managers in organizing measures to inform and influence platform staff.

Further analysis by simulation revealed that the effect of information provision reduces over time. Increasing the information provision period does not significantly affect time and risk perceptions Thus, information provision periods should be short and intensive. Furthermore, explaining why behavior/routines should change is more important than simply providing information. Experiences with dedicated intensive information sessions for introducing a new arrival procedure were positive.

\subsection{Managerial control and influence}

Reprimands by sector management can be an effective method to influence behavior of employees. Field study data however show that the probability for a team member 
to receive a reprimand from a sector manager for a specific action is low (Just culture [8]) The sensitivity analysis results also indicated a low importance of the reprimandrelated parameters. However, if the credibility intervals of the reprimand probability and severity include high values, their effect on motivation would increase considerably. Nevertheless, even in this case reprimands would impact extrinsically motivated behavior and would have only a short-term effect on motivation.

Since the probability for receiving reprimands from sector managers is low, other control options need to be explored. More frequent use of observers to check rule compliance on the platform could be considered. The experience with employees performing time measurements and monitoring the correct execution of the arrival procedure was positive. The benefit of using operational personal for these measurements and observations is twofold. First, important safety statistics for the management and safety departments can be collected. In the second place, the safety awareness of the observers and other employees can be improved.

\subsection{Social interaction in teams}

Team composition has a strong influence on the formation of desired and undesired norms and routines in teams. Expressive persons with a negative attitude towards safety can influence other team members in an undesirable manner. The negative effects are amplified throughout the whole department due to varying team compositions. Sector managers are considering the introduction of a registration system for management and team leaders to record undesired behaviour of employees. Such a system might help in identifying expressive employees with negative attitudes. Such identification would make it possible to rearrange teams to limit social contagion of undesired attitudes and behavior.

The results suggest enhancing the leadership capabilities of team leaders. Personal training of team leaders could improve their influence on team members.

\subsection{Autonomy}

Theory [7] suggests that autonomy is an important need influencing human motivation. Our sensitivity analysis revealed that the level of rule details has a significant influence on motivation. Employees argue that the operational performance goals cannot be met if all rules are followed, and argue for more freedom in the execution of their tasks. Furthermore, field study data indicated that the participation of employees in the rule formation process was limited. The results suggest that facilitating and stimulating feedback from the platform to the rule formation process by management would contribute positively to the autonomy need. Some researchers consider this process as a bottom-up formation of rule ownership by employees $[11,12]$

\section{Discussion and conclusions}

In this paper, a formal agent-based motivation model was presented, based on an integrated theoretical basis from social science. All the theories used for the model development were extensively validated by experiments with human subjects and received wide support. The validity of the expectancy theory has been extensively tested by empirical studies $[15,22]$. These studies have consistently found that expectancy type attitude measures are significantly correlated with measures of individual motivation and task performance. Self-determination theory, which is the basis of the model, is well accepted in social science. Its psychometric instruments are well validated [7]. A recent study described in [24] provides evidence that the psychometric properties of measures of motivation are aligned with self-determination theory. Self-determination theory has similarities with other influential theories of human motivation. In particular, [19] identifies four universal human motives: achievement, affiliation, power, and avoidance. Achievement is related to the competence need. Affiliation is similar to the relatedness need. The power motive is interpreted in [19] as having an impact on the world and reaching the sense of control. Furthermore, the power motive is to a degree reflected in the social contagion process, by which agents influence each other. In particular, the degree of influence might incorporate power aspects (e.g., based on the French \& Raven's framework [9]). The avoidance motive is not directly included in self-determination theory. However, in our model it may be related to the safety need. Furthermore, this motive is also reflected in social contagion between agents, as it determines the intensity and frequency of interaction through social contagion parameters.

In the study presented in the paper, a good agreement is demonstrated between the simulated results obtained using the proposed model and real data from the ground service organization under study. However, to gain more confidence in the model, more validation studies need to be performed. Data gathering for such studies is a time-consuming and difficult task, which on the one hand requires support of the organization, and on the other hand expertise in social science and ethnography. In our project, data were gathered by professional ethnographers.

A number of important individual, social, and organizational factors were identified by sensitivity analysis based on the model, which influence the motivation of the platform employees to comply with safety regulations. It is 
suggested that to achieve long lasting compliance, these factors need to be addressed by management. The sensitivity analysis identified the importance of social relations and norms for motivation and compliance. Other important findings concerned information provision by management to the employees. In particular, it is important not only to provide information, but also to explain the reasons for, and consequences of, rules and regulations.

In the paper, the compliance of employees to a specific task - FOD check - was investigated. However, the same modeling approach could also be used to study other tasks. Moreover, in a discussion with another ground service organization from a different national culture, findings from this study were recognized as relevant for their organization too. However, to determine the generality of the model-driven approach, more detailed studies at other organizations are required.

To the best knowledge of the authors, this is the first attempt to approach the problem of compliance in airline ground service organizations by a model-driven simulation study of the employees' motivation. Previous studies on safety occurrences at airline ground service organizations (e.g., [2]) mainly used statistical data analysis and informal identification of possible causes of these occurrences.

The formal agent-based modeling and simulation approach used here has a potential to become a useful decision support tool for managers at airline ground service organizations.

Acknowledgments We are grateful to Gert Jan van Hilten and Patrick Pronk for practical support of this research. Furthermore, we would like to thank David Passenier, dr. Robert Jan de Boer, and dr. Nicoletta Dimitrova for stimulating discussions in the course of this research. We thank prof.dr. Warren Walker for his valuable comments on the paper.

Open Access This article is distributed under the terms of the Creative Commons Attribution 4.0 International License (http:// creativecommons.org/licenses/by/4.0/), which permits unrestricted use, distribution, and reproduction in any medium, provided you give appropriate credit to the original author(s) and the source, provide a link to the Creative Commons license, and indicate if changes were made.

\section{Appendix A: The goals of a platform employee agent}

\section{G1.1 Achieve the highest time efficiency}

Handling all assigned aircraft on-time during the turnaround process can be seen as the main goal for platform staff during their normal daily operations. This deeply rooted goal can be clearly observed and was highlighted during the interviews with employees. Management targets support the performance driven mentality of platform staff.

Most employees have a long employment history and a high commitment and solidarity towards the company and their passengers. Satisfying passengers by avoiding delays in the turnaround process is deeply rooted in the minds of the operational staff. Competence goals are therefore strongly related to working as efficient as possible with respect to time. For years management have been giving time efficiency the highest priority. More recently the management priority has shifted towards safety as the main priority after a series of serious accidents and incidents. This priority shift is not entirely adapted by the operational personnel yet. A frequently heard comment to this is the following: "if we will comply with all (safety) rules, most aircraft would no longer depart on time ".

\section{G1.2 Prevent aircraft, equipment and/or infrastructural damage}

The platform environment can be hectic and busy during an aircraft turnaround process. Different companies and departments carry out their work within a short time period. Causing aircraft, equipment or infrastructural damage has a high impact on the organization, customers and employees. The consequences of aircraft damage in particular in terms of delay costs and maintenance costs can be high. Platform staff is well aware of the impact of incidents and avoiding damage has a high priority.

In the culture which is present at the workplace, involvement in incidents strongly affects the feeling of competence of platform staff. In most cases the involved ones need to report the damage to the management. The consequences are however limited for the employee since the management claims there is a just culture at the organization. In a just culture employees are not punished for actions, omissions or decisions, but gross negligence, willful violations and destructive acts are not tolerated.

\section{G2.1 Prevent personal injury}

Personal injury during operations is mainly caused by falling from height, moving equipment (collision) and exposure to environmental conditions (noise). These types of incidents can mostly be prevented by following rules and procedures, using physical safety barriers and wearing personal protective equipment (PPT). Occupational safety has a high management priority. Personnel indicated that returning home safely after work is a top priority. Based on low compliance levels to applicable rules and procedures a discrepancy between preventing personal injury and following rules seems to exist. 


\section{G3.1 Achieve high team acceptance}

The team acceptance goal is driven by the need of an individual to feel a sense of belonging to a group. The goal is satisfied when people feel appreciated and/or accepted by their colleagues. Following the team norms is a condition for acceptance. A social norm is based on reciprocal expectations of the people within the group. It is what people in some group believe to be normal, that is, believed to be a typical action, an appropriate action, or both [18]. Social norms can be stiffly resistant to change. The working paper by Unicef [18] provides some other ways of measuring social norms and was used as a guidance for the identification of the norms considered in this thesis.

The mutual relationships and dependencies between team members can be very strong. The field study revealed that maintaining good relationships with colleagues is an important job satisfaction requirement. In this respect, the team leader has a special role. The team leader is a member of a team, but has different responsibilities than the other team members. The team leader is a link between sector management and the workfloor. Sector management considers team leaders as the ambassadors of management norms at the workfloor. Team leaders struggle with the conflicting interests of management and workfloor. The result is the emergence of team leader norms, which are partly fulfilling the management norms and partly team norms.

\section{G3.2 Achieve high management acceptance}

Following rules and procedures, the management norms, are the condition for management acceptance. In general, the priority for management acceptance is much lower than the team acceptance priority for most team members. A relatively strong management aversion and incomprehension were identified during the data gathering phase. Complaints vary from inadequate working equipment to problems with holiday requests. This incomprehension strongly affects the influence relations between sector management and platform staff.

\section{G4.1 Achieve a high level of freedom in the execution of tasks}

The autonomy need in relation to platform operations is characterized by freedom in execution of tasks. Following rules and procedures restricts the freedom in execution of tasks. The number of rules and level of detail by which the process is described influence largely the flexibility in the operation execution. Also the amount of control is an important factor which can restrict the perceived freedom.

\section{G4.2 Achieve high psychological ownership of rules}

Ownership of rules is related to commitment and plays an important role in compliance with those rules, even in situations where no control exists. It is important to ensure that rules and procedures match the conditions at the workfloor. Team members and team leaders are the experts in carrying out the work and the rules and procedures should be in alignment with their (safety-related) beliefs. Autonomy is strongly affected by (new) rules which are not supported by platform staff. This frustration increases the incomprehension between management and operational personnel and affects negatively the feeling of autonomy.

\section{Appendix B: Model parameters}

In the table below Low (L) corresponds to [0, 0.33), Medium (M) corresponds to $[0.33,0.67)$, and High $(\mathrm{H})$ corresponds to $[0.67,1]$. For negative values of instrumentalities $\underline{L}$ stands for $[0,-0.33), \underline{\mathrm{M}}$ stands for $[-0.33,-0.67), \underline{\mathrm{H}}$ stands for $[-0.67,-1]$. Other intervals are specified by numbers.

\begin{tabular}{|c|c|c|c|c|}
\hline Param & Description & $\begin{array}{l}\text { Range in } \\
\text { 'Perform } \\
\text { FOD } \\
\text { check' } \\
\text { (option 1) }\end{array}$ & $\begin{array}{l}\text { Range in } \\
\text { 'Not perform } \\
\text { FOD check' } \\
\text { (option } 2 \text { ) }\end{array}$ & Explanation \\
\hline
\end{tabular}

\begin{tabular}{|c|c|c|c|c|}
\hline $\begin{array}{l}\text { E11 } \\
\text { E21 }\end{array}$ & $\begin{array}{l}\text { Belief about } \\
\text { the likelihood } \\
\text { that } \\
\text { performing } \\
\text { the action } \\
\text { would save } \\
\text { time }\end{array}$ & 0 & M & $\begin{array}{l}\text { Performing FOD check does } \\
\text { not save time. Not } \\
\text { performing the FOD check } \\
\text { saves a small amount of time } \\
\text { (approx. } 2-3 \text { minutes) for an } \\
\text { individual }\end{array}$ \\
\hline E12 & Belief about & M & 0 & Performing FOD will cost \\
\hline
\end{tabular}




\begin{tabular}{llll}
\hline Param Description & $\begin{array}{l}\text { Range in } \\
\text { 'Perform }\end{array}$ & $\begin{array}{l}\text { Range in } \\
\text { 'Not perform }\end{array}$ & Explanation \\
& FOD & FOD check' & \\
& check' & (option 2) & \\
& (option 1) & & \\
\end{tabular}

E13

E23

E14

E24

E15

E16 the likelihood

that

performing

the action

would cost

additional

time

Belief about

the likelihood

that the action

would result

in aircraft,

equipment or

infrastructural

damage

Belief about

the likelihood

that the action

would prevents

aircraft,

equipment or

infrastructural

damage

Belief about

the likelihood

that the action

would result

in personal

injury

Belief about

the likelihood

that the action

would prevent

personal

injury

Belief about the likelihood

that the agent is familiar with

the team norm

Degree of similarity between

the action and the team norm

Degree of difference between
0

M

M

0

$[0,0.2]$

$\mathrm{H}$

0

for high stress additional time (approx. 2-3

minutes) for an individual.

Not performing the FOD

check does not cost extra

time.

Most employees are aware

of the risks of vehicles,

cargo, FOD and other

objects on the VOP through

training and communication

with management/peers.

Observation of incidents and

visible damage is however

limited.

Most employees agree that

the FOD walk helps

preventing collision of

aircraft with objects on

VOP, engine damage due to

FOD and other incidents.

Experience with these kind

of incidents is however low.

Employees do not directly

see a relation with personal

injury. Highly unlikely that

debris will bounce back from

an engine fan blade.

Employees do not directly

see a relation with personal

injury. This relation is also

rarely observed in practice.

Only one team norm has been modeled in this study. The platform employees were well aware of this norm.

for high stress

conditions: 1

for other

conditions: 0

In the highly stressful situations the action 'Not perform FOD check' coincides with the team norm.

In the highly stressful situations the action 


\begin{tabular}{llll}
\hline Param Description & Range in & Range in & Explanation \\
& 'Perform & 'Not perform & \\
& FOD & FOD check' & \\
& check' & (option 2) & \\
& & \\
& (option 1) & & \\
\hline
\end{tabular}

\begin{tabular}{|c|c|c|c|c|}
\hline E28b & the action and the team norm & $\begin{array}{l}\text { conditions: } 0 \\
\text { for other } \\
\text { conditions: } 1\end{array}$ & & $\begin{array}{l}\text { 'Perform FOD check' contradicts the team } \\
\text { norm. }\end{array}$ \\
\hline $\begin{array}{l}\text { E19a } \\
\text { E29a }\end{array}$ & $\begin{array}{l}\text { Degree of similarity between } \\
\text { the action and the rule/procedure }\end{array}$ & 1 & 0 & $\begin{array}{l}\text { Degree of similarity equals one if the action } \\
\text { is carried out according to the rule/procedure. } \\
\text { Otherwise value is zero. }\end{array}$ \\
\hline $\begin{array}{l}\text { E19b } \\
\text { E29b } \\
\text { E111b } \\
\text { E211b }\end{array}$ & $\begin{array}{l}\text { Likelihood that the agent is } \\
\text { familiar with the rule/procedure }\end{array}$ & $\mathrm{H}$ & $\mathrm{H}$ & $\begin{array}{l}\text { Likelihood that the agent knows the } \\
\text { rule/procedure is high because most rules are } \\
\text { trained during education and refreshment trainings. } \\
\text { Furthermore important rules and procedures are } \\
\text { communicated frequently by management. }\end{array}$ \\
\hline $\begin{array}{l}\text { E110a } \\
\text { E210a }\end{array}$ & $\begin{array}{l}\text { Degree of difference between the } \\
\text { action and the rule/procedure }\end{array}$ & 0 & 1 & $\begin{array}{l}\text { Degree of difference equals } 1 \text { if the action is NOT } \\
\text { carried out according to the rule/procedure. If action } \\
\text { is carried out according to rule/procedure value } \\
\text { equals } 0 .\end{array}$ \\
\hline $\begin{array}{l}\text { E111 } \\
\text { E211 }\end{array}$ & $\begin{array}{l}\text { Belief about the likelihood that } \\
\text { the action would result in a } \\
\text { reprimand from a team member }\end{array}$ & 0 & {$[0,0.2]$} & $\begin{array}{l}\text { In most cases team members do not give reprimands } \\
\text { to each other for executing or not executing the FOD } \\
\text { check. Explicit task division increases the reprimand } \\
\text { probability. }\end{array}$ \\
\hline $\begin{array}{l}\text { E112 } \\
\text { E212 }\end{array}$ & $\begin{array}{l}\text { Belief about the likelihood that } \\
\text { the action would result in a } \\
\text { reprimand from a team leader }\end{array}$ & 0 & {$[0,0.2]$} & $\begin{array}{l}\text { Likelihood that team leader gives a reprimand for } \\
\text { executing or not executing the FOD check is low; it is } \\
\text { a reflection of just culture, which is established at the } \\
\text { organization. In general, the likelihood that a team } \\
\text { leader gives reprimands depends on the team leader's } \\
\text { type. Explicit task division increased the reprimand } \\
\text { probability significantly. }\end{array}$ \\
\hline $\begin{array}{l}\text { E113 } \\
\text { E213 }\end{array}$ & $\begin{array}{l}\text { Belief about the likelihood } \\
\text { that the action would result } \\
\text { in a reprimand from a } \\
\text { sector manager }\end{array}$ & 0 & {$[0,0.2]$} & $\begin{array}{l}\text { Likelihood that a team member receives reprimand } \\
\text { from sector manager is low; it is a reflection of just } \\
\text { culture, which is established at the organization. } \\
\text { Sector manager cannot observe the entire operation. }\end{array}$ \\
\hline $\begin{array}{l}\mathrm{I} 111 \\
\mathrm{I} 211\end{array}$ & $\begin{array}{l}\text { Belief about the likelihood } \\
\text { that a higher time efficiency } \\
\text { is reached by shortening the } \\
\text { total execution time }\end{array}$ & 0 & {$[0,0.2]$} & $\begin{array}{l}\text { If the team arrives on time at the gate (before the } \\
\text { aircraft arrives), executing or not executing the FOD } \\
\text { check does not significantly influence the total } \\
\text { execution time. Many platform employees indicated } \\
\text { no relation between these two states. }\end{array}$ \\
\hline $\begin{array}{l}\mathrm{I} 121 \\
\mathrm{I} 221\end{array}$ & $\begin{array}{l}\text { Belief about the likelihood } \\
\text { that time efficiency is } \\
\text { reduced due to the longer } \\
\text { total execution time }\end{array}$ & {$[0,-0.2]$} & 0 & $\begin{array}{l}\text { If the team arrives on time at the gate (before the } \\
\text { aircraft arrives), executing or not executing the FOD } \\
\text { check does not significantly influence the total } \\
\text { execution time. Many platform employees indicated } \\
\text { no relation between these two states. }\end{array}$ \\
\hline $\begin{array}{l}\mathrm{I} 131 \\
\mathrm{I} 231\end{array}$ & $\begin{array}{l}\text { Perceived severity of the } \\
\text { aircraft, equipment or } \\
\text { infrastructural damage }\end{array}$ & 0 & $\underline{\mathrm{M}}$ & $\begin{array}{l}\text { There is a general understanding that FOD can cause } \\
\text { engine damage. The severity of engine damage is } \\
\text { hazardous but not catastrophic. The severity of } \\
\text { damage in case of collisions is high. }\end{array}$ \\
\hline I141 & Perceived effectiveness & M & 0 & The extent to which the FOD walk can prevent damage \\
\hline
\end{tabular}




\begin{tabular}{llll}
\hline Param Description & Range in & Range in & Explanation \\
& 'Perform & 'Not perform & \\
& FOD & FOD check' & \\
check' & (option 2) & \\
& (option 1) & & \\
& & &
\end{tabular}

\begin{tabular}{|c|c|c|c|c|}
\hline I241 & $\begin{array}{l}\text { of preventing aircraft, } \\
\text { equipment or } \\
\text { infrastructural damage }\end{array}$ & & & $\begin{array}{l}\text { is limited. Small objects (debris) cannot be seen due to } \\
\text { time and physical limitations. }\end{array}$ \\
\hline $\begin{array}{l}\mathrm{I} 152 \\
\mathrm{I} 252\end{array}$ & $\begin{array}{l}\text { Perceived severity of the } \\
\text { personal injury }\end{array}$ & & $\underline{\mathrm{M}}$ & Severity of injury can be substantial. \\
\hline $\begin{array}{l}\mathrm{I} 162 \\
\mathrm{I} 262\end{array}$ & $\begin{array}{l}\text { Perceived effectiveness of } \\
\text { preventing personal injury }\end{array}$ & M & 0 & $\begin{array}{l}\text { Removing objects contributes towards avoiding } \\
\text { personal injury. }\end{array}$ \\
\hline $\begin{array}{l}\text { I173a } \\
\text { I273a }\end{array}$ & $\begin{array}{l}\text { Perceived importance of } \\
\text { following the team norm }\end{array}$ & M & M & $\begin{array}{l}\text { The norm considered in the case study is of medium } \\
\text { importance. }\end{array}$ \\
\hline $\begin{array}{l}\text { I183a } \\
\text { I283a }\end{array}$ & $\begin{array}{l}\text { Perceived contribution of } \\
\text { not following the team } \\
\text { norm to team acceptance }\end{array}$ & $\underline{\mathrm{M}}$ & $\underline{\mathrm{M}}$ & $\begin{array}{l}\text { The norm considered in the case study is of medium } \\
\text { importance. }\end{array}$ \\
\hline $\begin{array}{l}\text { I193a } \\
\text { I293a }\end{array}$ & $\begin{array}{l}\text { Perceived importance of } \\
\text { following the rule/procedure }\end{array}$ & M & M & $\begin{array}{l}\text { This parameter indicates how important it is to follow } \\
\text { the rules to be accepted by management. Most } \\
\text { employees understand the purpose of the FOD check. } \\
\text { Some employees indicate the FOD check is important } \\
\text { while others indicate it is not really important for them. }\end{array}$ \\
\hline $\begin{array}{l}\text { I1103a } \\
\text { I2103a }\end{array}$ & $\begin{array}{l}\text { Perceived contribution of } \\
\text { not following the } \\
\text { rule/procedure to } \\
\text { management acceptance }\end{array}$ & $\underline{\mathrm{M}}$ & $\underline{\mathrm{M}}$ & $\begin{array}{l}\text { The FOD check procedure considered in the case study } \\
\text { is of medium importance for the platform employees. }\end{array}$ \\
\hline $\begin{array}{l}\text { I1 } 113 \\
\text { I } 2113\end{array}$ & $\begin{array}{l}\text { Relation between team } \\
\text { member reprimand and } \\
\text { team acceptance (perceived } \\
\text { severity) }\end{array}$ & 0 & {$[0,-0.2]$} & $\begin{array}{l}\text { From field observations and interviews it became clear } \\
\text { that for most people the effect of reprimands received } \\
\text { from a fellow team member is low or not felt at all. }\end{array}$ \\
\hline $\begin{array}{l}\text { I1123 } \\
\text { I } 2123\end{array}$ & $\begin{array}{l}\text { Relation between team } \\
\text { leader reprimand and team } \\
\text { acceptance (perceived } \\
\text { severity) }\end{array}$ & 0 & {$[0,-0.2]$} & $\begin{array}{l}\text { Field observations and interviews revealed that the } \\
\text { severity of a reprimand from a team leader is low. Team } \\
\text { leaders do not have the coercive power to punish team } \\
\text { members. Severity of reprimand also depends on team } \\
\text { leader type. }\end{array}$ \\
\hline $\begin{array}{l}\text { I1133 } \\
\text { I2133 }\end{array}$ & $\begin{array}{l}\text { Relation between sector } \\
\text { manager reprimand and } \\
\text { management acceptance } \\
\text { (perceived severity) }\end{array}$ & 0 & $\underline{\mathrm{L}}$ & $\begin{array}{l}\text { Severity of a reprimand from management is low. In } \\
\text { general employees know that reprimands do not have } \\
\text { severe consequences. Coercive power of management is } \\
\text { relatively low. Repeated reprimands can however have } \\
\text { larger consequences but this is not incorporated in the model. }\end{array}$ \\
\hline V1 & $\begin{array}{l}\text { Importance of achieving } \\
\text { a high level of competence }\end{array}$ & {$[0.6,0.8]$} & {$[0.6,0.8]$} & $\begin{array}{l}\text { Competence need is very important. Behavior is performance } \\
\text { driven. }\end{array}$ \\
\hline V2 & $\begin{array}{l}\text { Importance of achieving a } \\
\text { high level of occupational } \\
\text { safety }\end{array}$ & {$[0.2,0.4]$} & {$[0.2,0.4]$} & Priority for personal safety at the work floor is relatively low. \\
\hline V3 & $\begin{array}{l}\text { Importance of maintaining } \\
\text { a sense of belonging and }\end{array}$ & {$[0.6,0.8]$} & {$[0.6,0.8]$} & $\begin{array}{l}\text { Keeping social relationships with colleagues is very important } \\
\text { for most employees. }\end{array}$ \\
\hline
\end{tabular}




\begin{tabular}{|c|c|c|c|c|}
\hline Param & Description & $\begin{array}{l}\text { Range in } \\
\text { 'Perform } \\
\text { FOD } \\
\text { check' } \\
\text { (option 1) }\end{array}$ & $\begin{array}{l}\text { Range in } \\
\text { 'Not perform } \\
\text { FOD check' } \\
\text { (option 2) }\end{array}$ & Explanation \\
\hline V4 & $\begin{array}{l}\text { Importance of striving for } \\
\text { control over own behavior and goals }\end{array}$ & {$[0.3,0.5]$} & {$[0.3,0.5]$} & The autonomy goals have a medium priority. \\
\hline IF1 & Amount of monitoring and control & $\mathrm{L}$ & $\mathrm{L}$ & $\begin{array}{l}\text { The perceived amount of monitoring and control } \\
\text { of sector management is low. Monitoring by } \\
\text { team members and team leader is high but there } \\
\text { is still freedom to perform the work the way the } \\
\text { employee prefers. Control by team members and } \\
\text { team leader is however very limited. This can also } \\
\text { be seen in the reprimand likelihood. Therefore, } \\
\text { outside control periods the amount of monitoring } \\
\text { and control is low. During control periods this } \\
\text { value is increased. }\end{array}$ \\
\hline $\mathrm{IF} 2$ & Level of rule details & M & M & $\begin{array}{l}\text { This factor is related to the perception of } \\
\text { employees that there are many rules which need } \\
\text { to be followed during normal operations. Field } \\
\text { research clarified that (particularly) experienced } \\
\text { employees indicate that following all rules } \\
\text { jeopardizes efficient operations. Another aspect } \\
\text { is related to the practicability of rules which were } \\
\text { initiated by managers without consulting the } \\
\text { professionals on the platform. }\end{array}$ \\
\hline IF3 & $\begin{array}{l}\text { Participation in the rule formation } \\
\text { process }\end{array}$ & 0 & 0 & $\begin{array}{l}\text { Participation in the formation and introduction } \\
\text { of new rules is very limited. }\end{array}$ \\
\hline w11 & $\begin{array}{l}\text { Importance of time efficiency for } \\
\text { the competence level }\end{array}$ & {$[0.7,0.9]$} & {$[0.7,0.9]$} & Expert opinion. \\
\hline w12 & $\begin{array}{l}\text { Importance of preventing damage } \\
\text { to aircraft, equipment and/or } \\
\text { infrastructure to the competence level }\end{array}$ & {$[0.5,0.7]$} & {$[0.5,0.7]$} & Expert opinion. \\
\hline w21 & $\begin{array}{l}\text { Importance of preventing personal } \\
\text { injury to the occupational safety level }\end{array}$ & {$[0.4,0.6]$} & {$[0.4,0.6]$} & Expert opinion. \\
\hline w31 & $\begin{array}{l}\text { Importance of team acceptance to the } \\
\text { sense of belonging and attachment to } \\
\text { colleagues }\end{array}$ & {$[0.8,1]$} & {$[0.8,1]$} & Expert opinion. \\
\hline w32 & $\begin{array}{l}\text { Importance of management acceptance } \\
\text { to the sense of belonging and } \\
\text { attachment to colleagues }\end{array}$ & {$[0,0.2]$} & {$[0,0.2]$} & Expert opinion. \\
\hline w41 & $\begin{array}{l}\text { Importance of freedom in execution } \\
\text { to the control over own behavior and } \\
\text { goals }\end{array}$ & {$[0.4,0.6]$} & {$[0.4,0.6]$} & Expert opinion. \\
\hline w42 & $\begin{array}{l}\text { Importance of psychological ownership } \\
\text { of rules to the control over own } \\
\text { behavior and goals }\end{array}$ & {$[0.4,0.6]$} & {$[0.4,0.6]$} & Expert opinion. \\
\hline
\end{tabular}




\section{Appendix C: Sensitivity analysis results}

The following table provides a list with the most important model parameters in the order of decreasing importance obtained by the sensitivity analysis with the parameter ranges as defined in Appendix B.

\begin{tabular}{llr}
\hline Param. & Explanation & $d\left(x_{i}\right)$ \\
\hline I141 & $\begin{array}{l}\text { Perceived effectiveness of preventing aircraft, equipment or } \\
\text { infrastructural damage } \\
\text { Belief about the likelihood that time efficiency is reduced due to } \\
\text { the longer total execution time } \\
\text { Influence relation between management and team } \\
\text { members/leaders }\end{array}$ & 0.6 \\
I110b & Perceived effectiveness of preventing personal injury & 0.25 \\
& Agent's openness & 0.15 \\
I162 & Level of rule details & 0.15 \\
$\alpha$ params & Perceived importance of following the rule/procedure & 0.13 \\
IF2 & Perceived severity of the personal injury & 0.10 \\
I110a & Agent's expressiveness & 0.06 \\
I152 & Likelihood that the agent is familiar with the rule/procedure & 0.05 \\
$\varepsilon$ params & Belief about the likelihood that performing the action would cost & 0.04 \\
E19b & additional time & 0.03 \\
E12 & Belief about the likelihood that the action would prevents aircraft, & 0.03 \\
E14 & equipment or infrastructural damage & 0.03 \\
& & \\
\hline
\end{tabular}

The next table provides a list with the most important model parameters obtained by the sensitivity analysis with the decision making parameters (expectancies, instrumentalities, and valences) ranging in $(0,1)$.

\begin{tabular}{llc}
\hline Param. & Explanation & $d\left(x_{i}\right)$ \\
\hline I141 & $\begin{array}{l}\text { Perceived effectiveness of preventing aircraft, equipment or } \\
\text { infrastructural damage } \\
\text { I121 }\end{array}$ & 0.52 \\
& $\begin{array}{l}\text { Belief about the likelihood that time efficiency is reduced due to } \\
\text { the longer total execution time } \\
\text { Influence relation between management and team } \\
\text { members/leaders }\end{array}$ & 0.37 \\
I162 & Perceived effectiveness of preventing personal injury & 0.25 \\
E111 & Belief about the likelihood that the action would result in a reprimand & 0.15 \\
& from a team member & 0.15 \\
E112 & Belief about the likelihood that the action would result in a reprimand & \\
& from a team leader & 0.14 \\
$\alpha$ params & Agent's openness & \\
IF2 & Level of rule details & 0.1 \\
I1113 & Relation between team member reprimand and team acceptance \\
(perceived severity) & 0.1 \\
I1123 & Relation between team leader reprimand and team acceptance & 0.1 \\
(perceived severity) & Perceived importance of following the rule/procedure & 0.1 \\
I110a & Perceived severity of the personal injury & 0.1 \\
\hline
\end{tabular}


$\varepsilon$ params

$\mathrm{E} 19 \mathrm{~b}$

E12

E14
Agent's expressiveness

0.08

Likelihood that the agent is familiar with the rule/procedure

0.08

Belief about the likelihood that performing the action would cost

0.07 additional time

Belief about the likelihood that the action would prevents aircraft, equipment or infrastructural damage

\section{Appendix D: What-if simulation outcomes for larger teams}

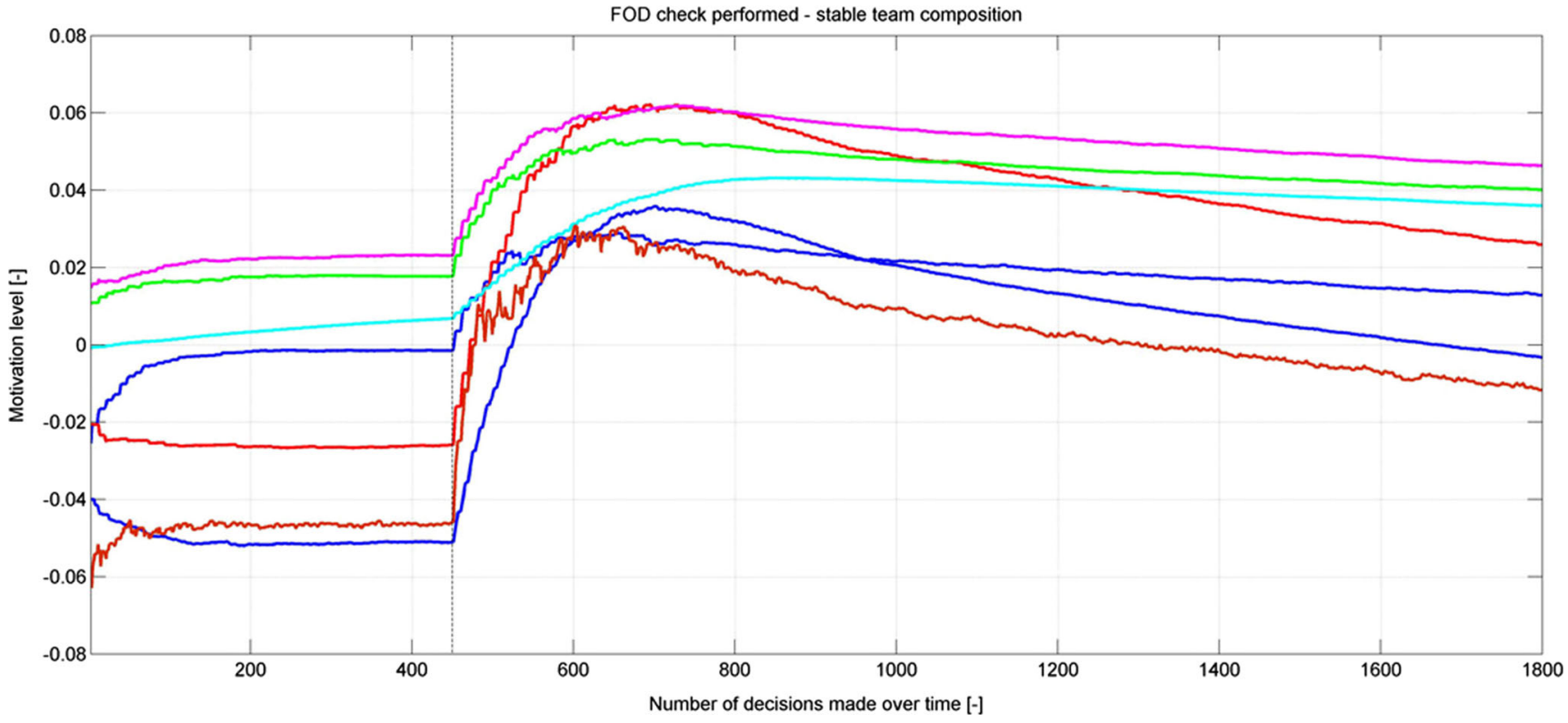

Fig. 7 The motivational forces of 7 agents in a team to perform FOD check in the three periods of the scenario under consideration

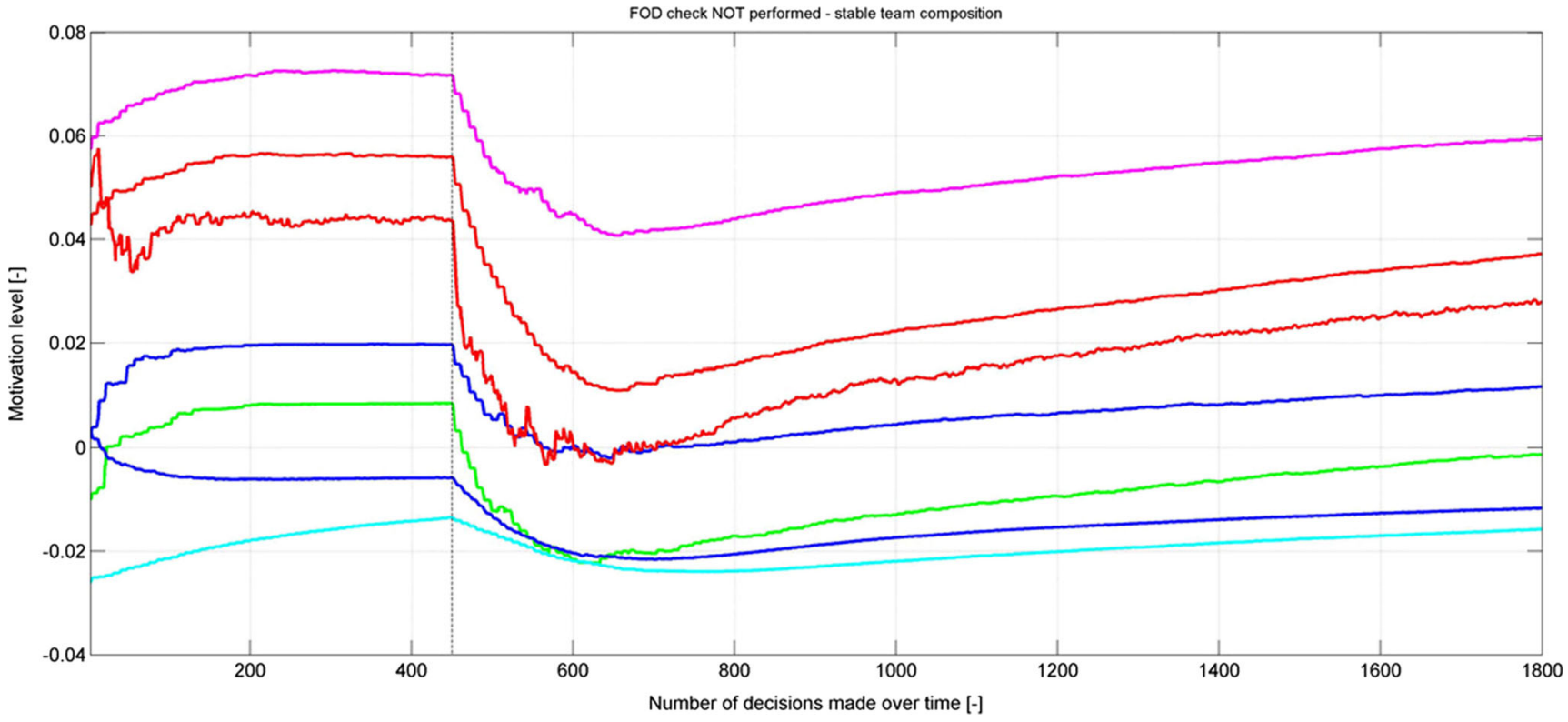

Fig. 8 The motivational forces of 7 agents in a team to not perform FOD check in the three periods of the scenario under consideration 


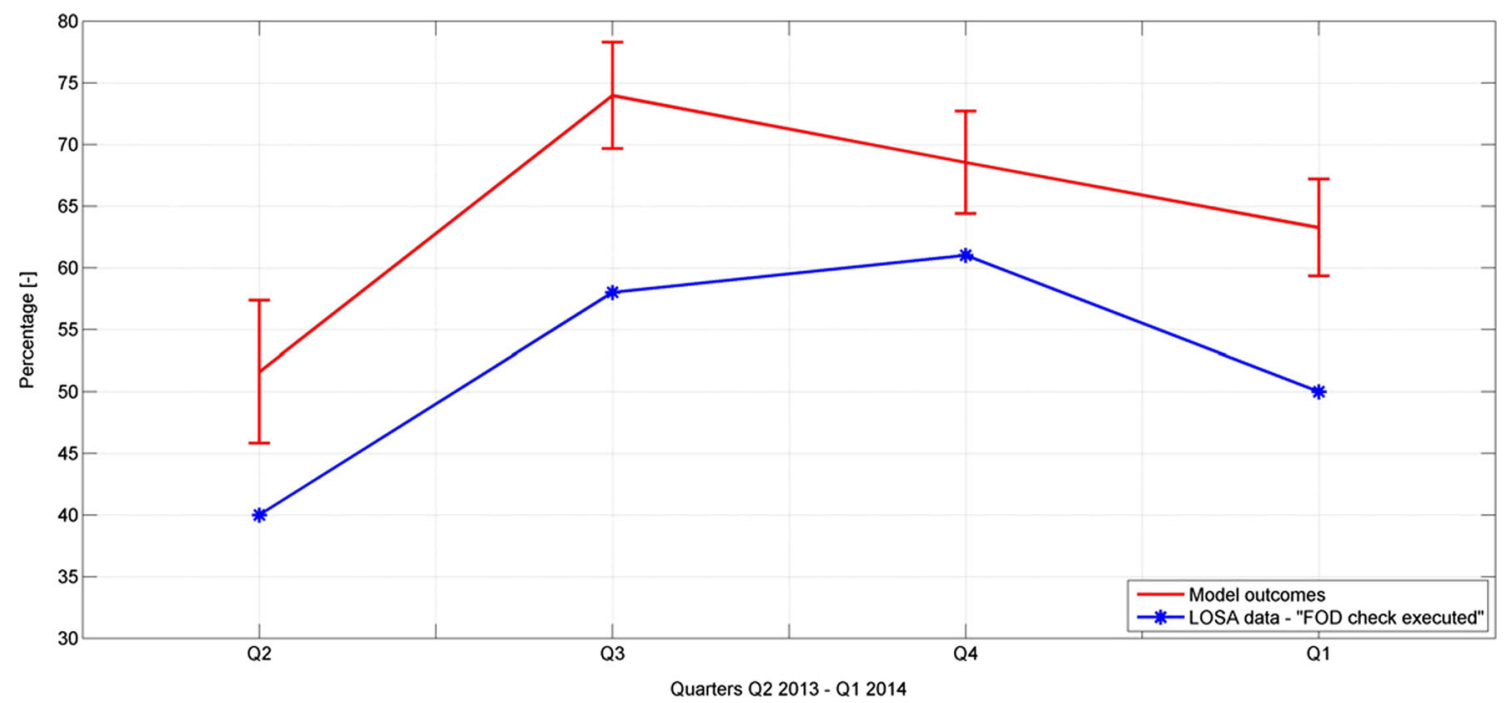

Fig. 9 The FOD check execution statistics (in \%) obtained from the ramp LOSA data and from the simulated model with teams of 7 agents

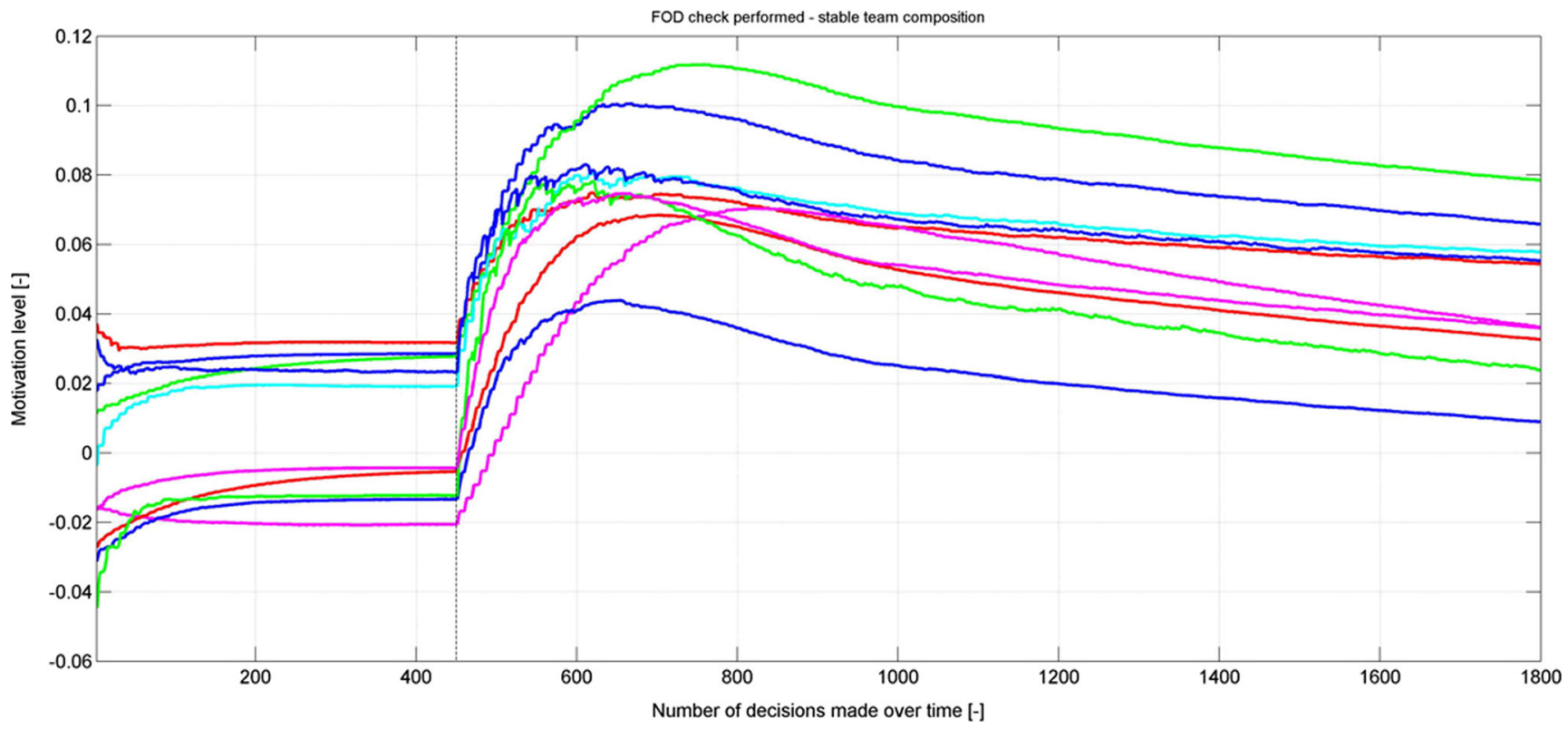

Fig. 10 The motivational forces of 10 agents in a team to perform FOD check in the three periods of the scenario under consideration 


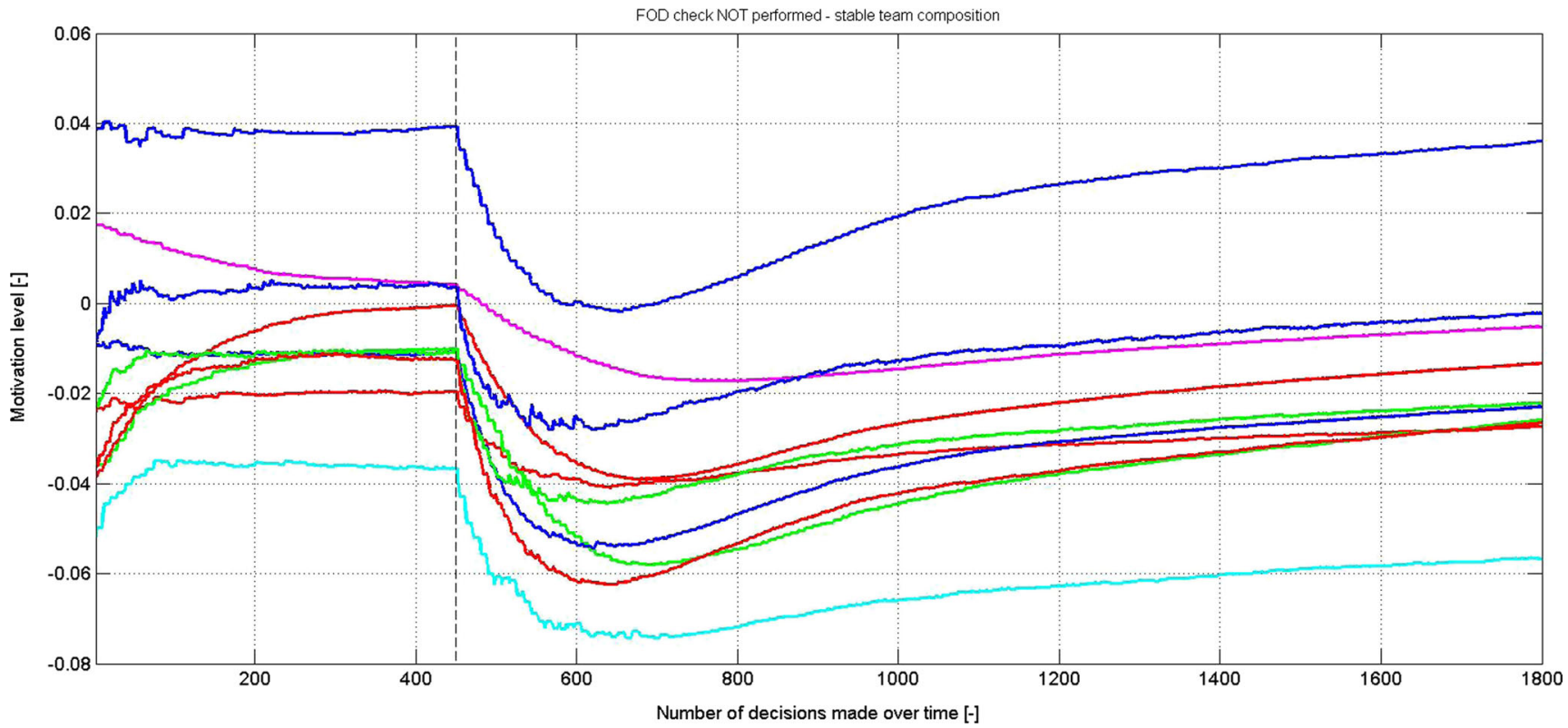

Fig. 11 The motivational forces of 10 agents in a team to not perform FOD check in the three periods of the scenario under consideration

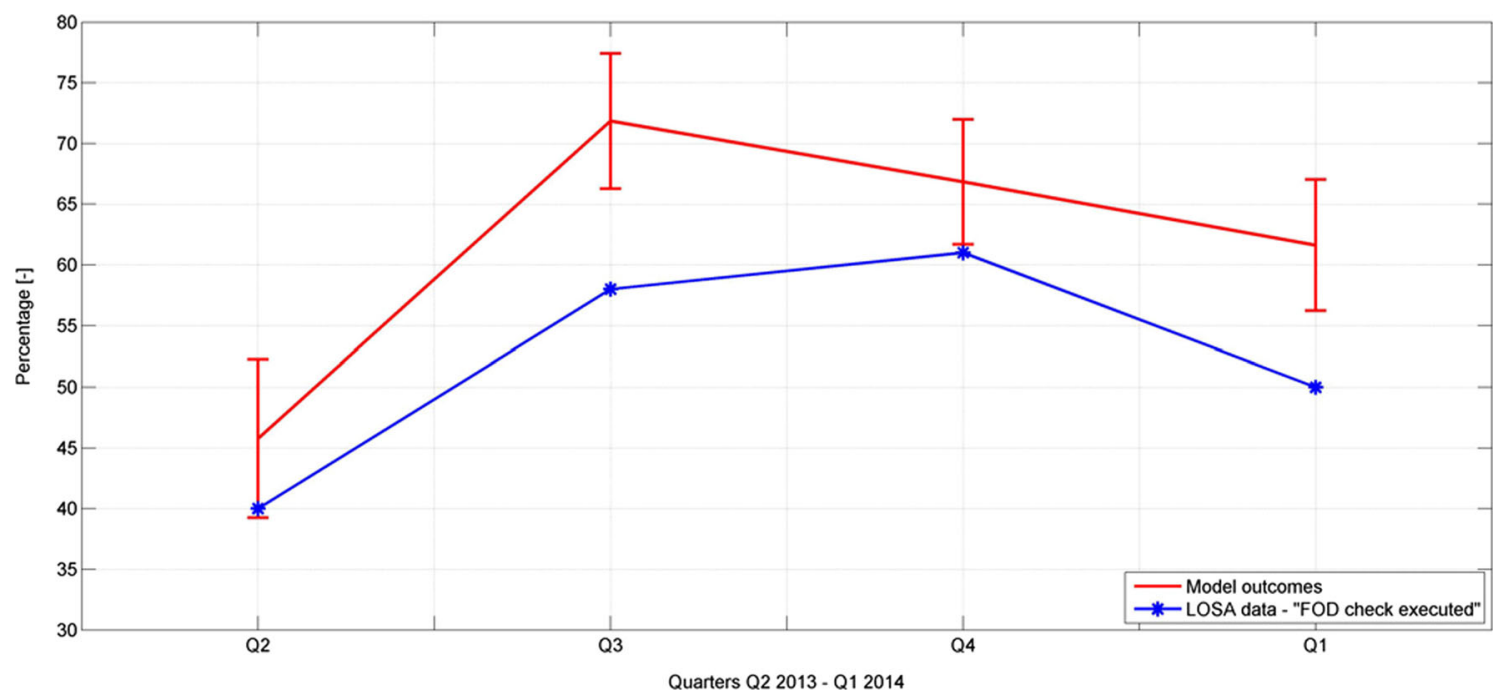

Fig. 12 The FOD check execution statistics (in \%) obtained from the ramp LOSA data and from the simulated model with teams of 10 agents 


\section{References}

1. Axelrod R (1997) The dissemination of culture a model with local convergence and global polarization. J Confl Resolut 41:2023-226

2. Balk A (2008) Safety of ground handling (Research Report No. NLR-CR-2007-961). Retrieved from EASA website: http://easa.europa.eu/essi/ecast/wpcontent/uploads/2011/08/ NLR-CR-2007-961.pdf:NationalAerospaceLaboratory

3. Bakker J (2014) Making sense of safety at KLM Ground Services. Master Thesis, VU University Amsterdam

4. Boeing (1998) Foreign object debris and damage prevention. Retrieved from http://www.boeing.com/commercial/ aeromagazine/aero01/textonly/s01txt.html

5. de Boer RJ, de Jong S (2014) Application of STAMP to Facilitate Interventions to Improve Platform Safety. In: Proceedings of the 3rd STAMP workshop, boston

6. de Boer RJ, Koncak B, Habekotté R, Van Hilten GJ (2011) Introduction of ramp-LOSA at KLM Ground Services. In: Human factors of systems and technology, human factors and ergonomics society annual meeting. Shaker publishing, Maastricht

7. Deci EL, Vansteenkiste M (2004) Self-determination theory and basic need satisfaction: Understanding human development in positive psychology. Ricerche di Psichologia, 27, pp 17-34

8. Dekker S (2008) Just culture Balancing safety and accountability. Ashgate Publishing, Burlington

9. French J, Raven B (1959) The bases of social power. In: Cartwright D (ed) Studies in social power. Institute for Social Research, Ann Arbor, pp 150-167

10. Haest R (2014) Modeling and Analysis of Rule and Procedure Compliance in Airline Ground Operations: A multidisciplinary safety study at KLM Ground Services Master Thesis Delft University of Technology. (available from TU Delft repository)

11. Hale A (2013a) Working to rule, or working safely? Part 1: A state of the art review. Saf Sci 55:207-221

12. Hale A (2013b) Working to rule or working safely? Part 2: The management of safety rules and procedures. Saf Sci 55:222-231

13. Hegselmann R, Krause U (2002) Opinion dynamics and bounded confidence: models, analysis and simulation. J of Artificial Societies and Social Simulation 5:3
14. Heimering R (2014) Understanding the organizational routines of the KLM Ground Services handling staff regarding to personal safety rules/procedures Master Thesis. VU University, Amsterdam

15. Heneman HG, Schwab DP (1972) Evaluation of research on expectancy theory predictions of employee performance. Psychol Bull 78(1):1

16. Kahneman D (2011) Thinking, fast and slow New York Farrar Straus and Giroux

17. Kleijen JPC (1999) Validation of models: statistical techniques and data availability. Simulation Conference Proceedings, 1999 Winter. Vol 1. IEEE

18. Mackie G, Moneti F, Denny E, Shakya H (2012) What are Social Norms? How are They Measured? In: Working paper UNICEF / UCSD center on global justice poject cooperation agreement

19. McClelland DC (1987) Human motivation. CUP Archive

20. Mitleton-Kelly E (2013) Co-evolution of Intelligent Sociotechnical Systems Modelling and Applications in Large Scale Emergency and Transport Domains. Springer

21. Monti A, Soda G (2014) Perceived Organizational Identification and Prototypicality as Origins of Knowledge Exchange Networks. In: Brass DJ, Labianca G(JOE), Mehra A, Halgin DS, Borgatti SP (eds) Contemporary Perspectives on Organizational Social Networks (Research in the Sociology of Organizations Volume 40). Emerald Group Publishing Limited, pp 357-379

22. Pinder CC (1998) Work motivation in organizational behavior. Prentice-hall, NJ

23. Popova V, Sharpanskykh A (2011) Formal modelling of organisational goals based on performance indicators. Data Knowl Eng 70(4):335-364

24. Sebire SJ, Jago R, Fox KR, Edwards MJ, Thompson JL (2013) Testing a self-determination theory model of children's physical activity motivation: a cross-sectional study. Int J Behav Nutr Phys Act 10(1):111

25. Saltelli A, Tarantola S, Campolongo F, Ratto M (2004) Sensitivity Analysis In Practice: A Guide to Assessing Scientific Models. Wiley, West Sussex

26. Sharpanskykh A, Stroeve S (2011) An Agent-based Approach for Structured Modeling, Analysis and Improvement of Safety Culture. Computational and Mathematical Organization Theory 17:77-117 